\title{
A complete design of ducted propellers using the new computer system
}

\author{
Tadeusz Koronowicz, Prof. \\ Zbigniew Krzemianowski, Ph. D. \\ Teresa Tuszkowska, Ph. D. \\ The Szewalski Institute of Fluid Flow Machinery \\ of the Polish Academy of Sciences in Gdansk \\ Jan A. Szantyr, Prof. \\ Gdansk University of Technology
}

\begin{abstract}
The computer system for the completed design of the ducted ship propellers has some common blocks and procedures with the analogical system for open propellers that has already been presented in detail in the Polish Maritime Research [1]. This article describes only these blocks and procedures which are specific for the design of ducted propellers. These new blocks concern first of all the procedures for the design calculation of ducted propellers and for the analysis of the ducted propeller operation in the non-uniform velocity field behind the ship hull. The comparative analysis of computation results for different types of ducts is also presented.
\end{abstract}

Keywords: ship propellers; ducted propellers; design methods; computational fluid dynamics

\section{INTRODUCTION}

The design of ducted propellers is based on the same requirements and assumptions that were used in the design of open propellers $[1,6]$. The design procedure should lead to the compromise selection of the number and geometry of the propeller blades in order to ensure:

$\star$ the appropriate strength of the propeller blades

$\star$ the highest possible propulsive efficiency

$\star$ the absence of cavitation or only its limited presence, not leading to erosion, vibration and noise

$\star$ the acceptable level of propeller-induced pressure pulses on the hull

$\star$ the acceptable level of the unsteady bearing forces

The presence of the duct leads to the additional requirements:

$\star$ the appropriate selection of the duct geometry

$\star$ computation of the thrust generated by the duct

$\star$ computation of the velocity field generated by the duct at the propeller, both for the propeller design and for the analysis of propeller operation in the non-uniform velocity field behind the ship hull.

Similarly as in the case of an open propeller, the complete design system for the ducted propellers should incorporate 3 interacting programs (i.e. 3 blocks of procedures):

* programs for the determination of the design velocity field
- programs for the design of ducted propellers

* programs for the analysis of the ducted propellers operation in the non-uniform velocity field.

Moreover, the system should include the databases and graphical procedures for the selection of the duct geometry and procedures for calculation of the hydrodynamic forces and the velocity field generated by the duct. With reference to the system for open propellers design, the ducted propellers design system is supplemented with the following components:

is graphical presentation of the available duct geometries in order to facilitate duct selection

is procedures for determination of the design velocity field and of the axial hydrodynamic force generated by the duct

is procedure for the duct-induced modification of the velocity field on input to the program UNCA

is procedure for determination of the pressure pulses generated by the propeller around the duct-propeller system (based on the program UNCA)

is program for calculation of the pressure pulses generated by the duct (based on the program DUNCAN).

The appropriate interaction between the respective programs and procedures, supplemented with the above listed components, enables the complete design calculation of the ducted propeller. The presented program incorporates all necessary components integrated into one system and the design calculation may be controlled directly from the computer 
keyboard, without preparation of the separate input data files for different programs and procedures.

Moreover, the system includes several graphical procedures for control of the input data and of the intermediate results, as well as procedures for modification of the designed propeller geometry directly from the computer screen.

The block diagram of the computer system is similar to that for the open propellers, with the above described modifications taking into account the presence of the duct (cf. Fig. 1).

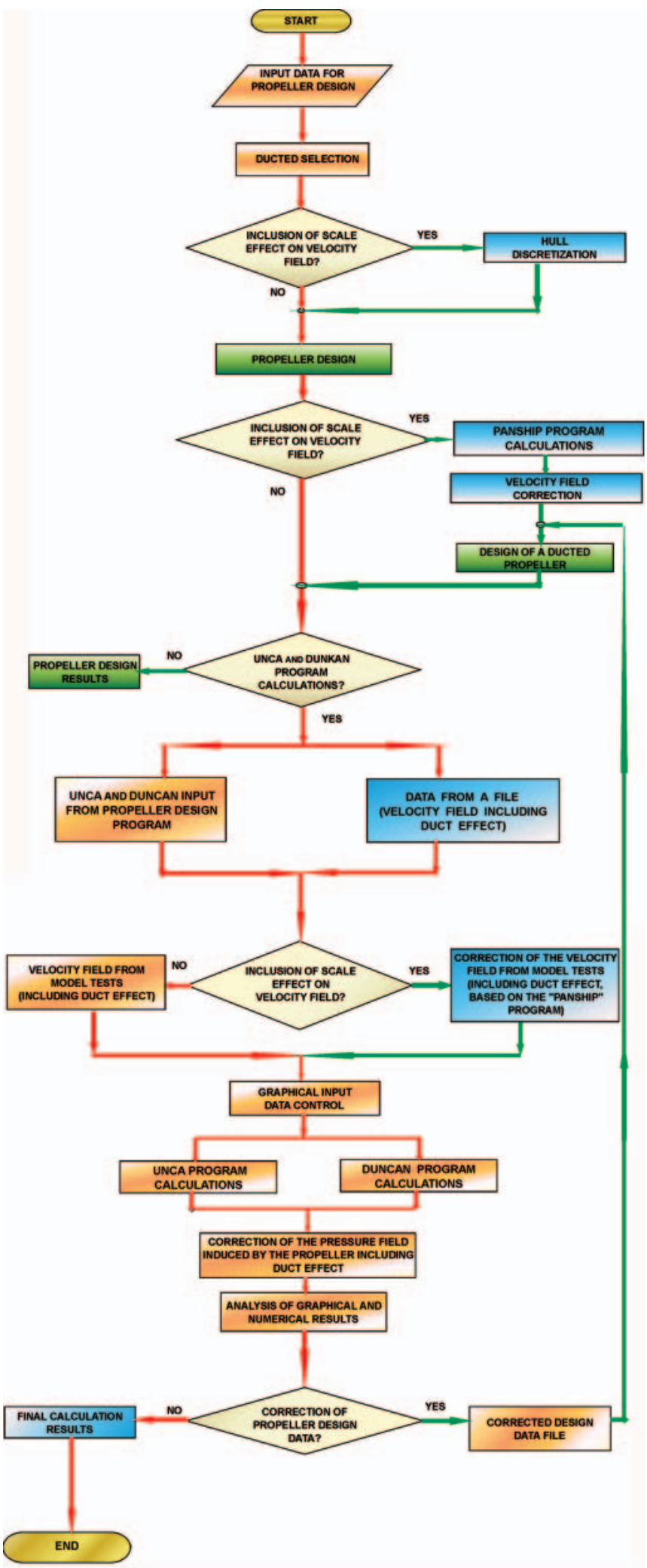

Fig. 1. The block diagram of the computer system for design of ducted propellers

\section{PRESENTATION OF THE SELECTED BLOCKS OF THE DESIGN SYSTEM}

This design system for design of ducted propellers incorporates several blocks similar to those of the system for design of open propellers. Only new blocks, specific for the case of ducted propellers are presented in detail in this article. Blocks common for both systems are only briefly mentioned.

\section{The input data}

The input data include all parameters necessary for initiation of one of four possible variants of the design calculation, analogically as for the open propellers. The input data may be introduced either in the form of a data file or directly from the computer keyboard. The program allows for graphical control of the introduced input data (e.g. the propeller blade outline, the distribution of the blade thickness, the radial distribution of the circumferentially averaged inflow velocity etc.). In the program for design of ducted propellers the selection of the duct type is the crucial problem. In this program one of the five available types of ducts may be selected.

\section{Ducts of technologically simple geometry}

Fig. 2 shows the cross - section of one of the geometrically simple ducts. The length $\mathrm{L}$ of this duct is equal to half of the propeller diameter. The gap between the propeller blade tip and the inner surface of the duct is equal to $1.0-1.5$ per cent of the propeller diameter. The geometrically simple ducts differ from one another in their thickness S. One of four values of $\mathrm{S}$ may be selected, numbered accordingly as No. 10, No. 13, No. 16 and No. 19. The exit angles $\alpha$ are different for different duct thickness, their optimum values have been determined experimentally.

Duct No. 10 DU10

Duct No. 13 DU13

$\mathrm{S} / \mathrm{L}=0.1333 \alpha=3 \mathrm{deg}$

Duct No. 16 DU16

$\mathrm{S} / \mathrm{L}=0.1733 \alpha=5 \mathrm{deg}$

Duct No. 19 DU19

$\mathrm{S} / \mathrm{L}=0.2133 \alpha=7 \mathrm{deg}$

$\mathrm{S} / \mathrm{L}=0.2533 \alpha=9 \mathrm{deg}$

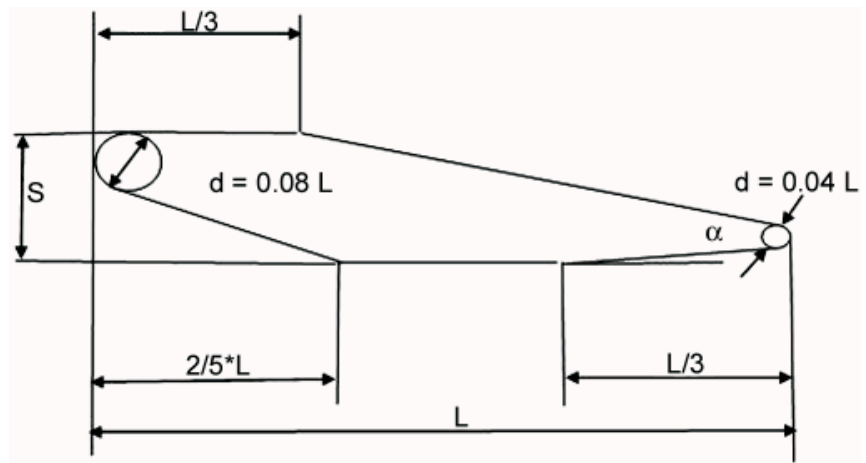

Fig. 2. The geometry of the technologically simple ducts

The duct DU19 has the most similar performance to the duct Wageningen 19A - the DU19 duct thrust generated in the bollard condition is only slightly smaller, but the performance at higher advance coefficients is better than that of the 19A. This is the consequence of the smaller drag of the duct DU19 due to the better flow conditions on the outside surface of the duct.

\section{Duct Wageningen 19A}

The geometry of the Wageningen 19A duct is partly simplified in comparison to other Wageningen ducts, therefore 
Tab. 1. The ordinates of the profile of the Wageningen 19A duct

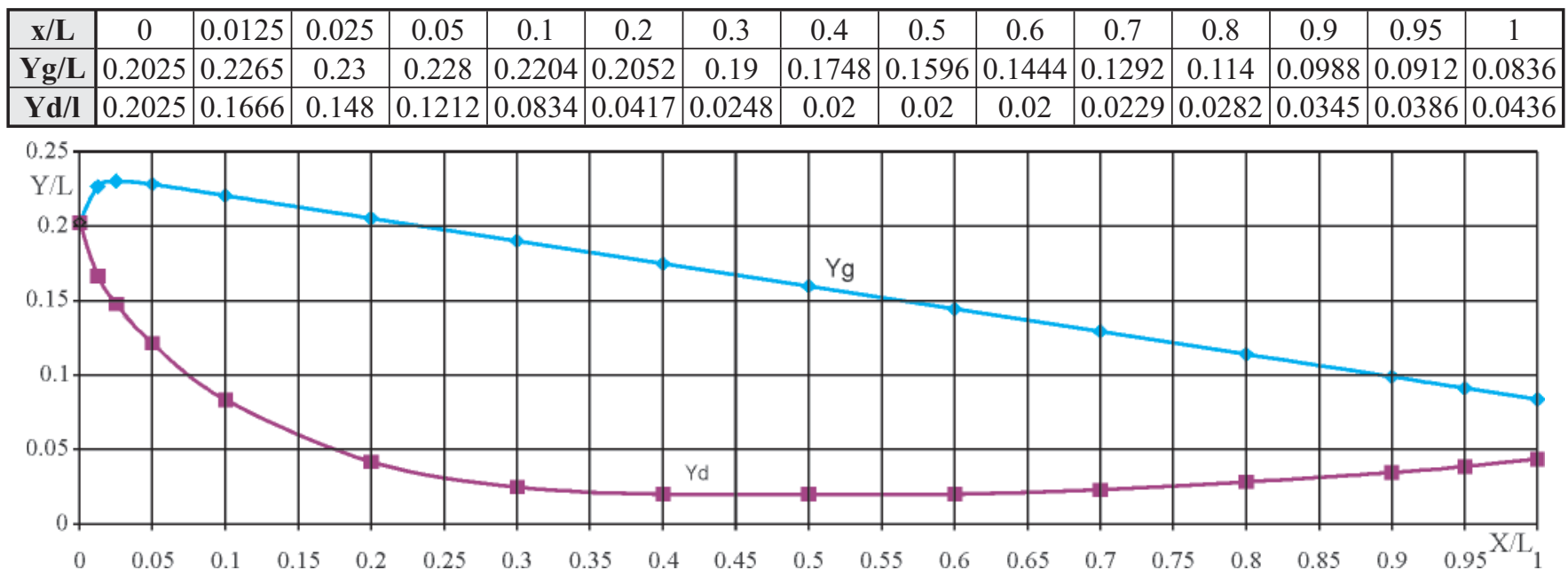

Fig. 3. The geometry of the Wageningen $19 \mathrm{~A}$ duct

this duct is the most frequently used one. Similarly as above, the duct length $\mathrm{L}$ is equal to half of the propeller diameter. The gap between the propeller blade tip and the inner surface of the duct is equal to 1 per cent of the propeller diameter. The non-dimensional ordinates of the duct profile (related to the duct length) are given in Tab. 1.

\section{The design program}

The algorithm for design of ducted propellers differs from the algorithm for design of open propellers presented in [1] only in a few details. The program based on this algorithm has been used for many years and it is thoroughly verified. The differing details are:

$\Rightarrow$ the calculations are conducted only for a given value of the total thrust

$\Rightarrow$ the program automatically divides the total thrust into parts generated by the propeller and by the duct. The part generated by the duct depends on the selected duct geometry and on the duct loading coefficient

$\Rightarrow$ the inflow velocity field at the propeller is corrected by including the velocity field induced by the duct.

The missing possibility for calculating the ducted propeller for a given power (available in design of open propellers) may be balanced by performing calculations for several values of thrust and attaining the required power in an iterative process. In the case of open propellers such an iterative process is performed automatically.

The design calculation of a ducted propeller is performed always in the same way, irrespective of the selected version of calculations. This calculation ends with the results presented in the form of a numerical file describing the designed propeller and corresponding drawings on the computer screen (the drawings may be printed and/or stored in computer memory). Fig. 4 shows an example of such a drawing, which may be viewed on the screen from arbitrarily selected angles and the most interesting view may be designated for printing.

\section{The program for the analysis of propeller operation in the non-uniform velocity field}

The computer program UNCA for the analysis of the propeller operation in the non-uniform velocity field is a very important part of the system. The crucial part of this program is the determination of the extent and intensity of different

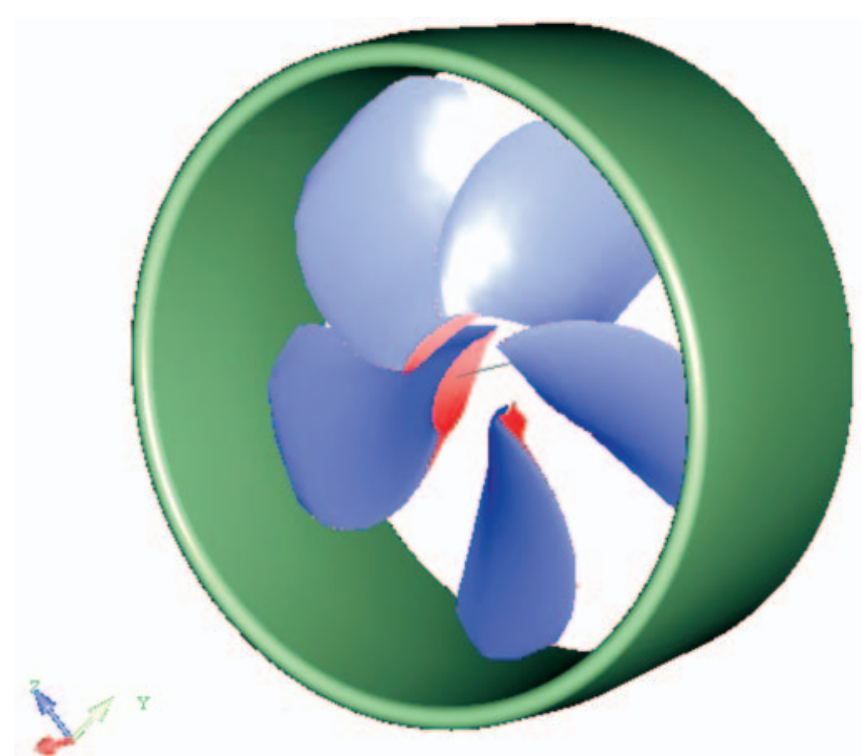

Fig. 4. The view of the designed ducted propeller

forms of cavitation on the blades of the propeller operating in the non-uniform inflow field. The original theoretical model integrates the unsteady vortex lifting surface theory with the unsteady sheet cavity theory. The detailed description of this algorithm is presented in $[4,5]$. Additionally, the program DUNCAN [6] for calculation of the unsteady pressure pulses generated by the duct was included in the system.

The newly designed ducted propeller is checked by means of the UNCA and DUNCAN programs from the point of view of:

$\Rightarrow$ the detection of different forms of cavitation in different angular position of the propeller blades in the ship hull wake

D the values of the pressure pulses generated on the hull surface or in the surrounding space. These pressure pulses are composed of these generated by the propeller (program UNCA) and by the duct (program DUNCAN)

$\rightarrow$ the values of unsteady bearing forces and moments.

After analyzing the above results the appropriate modifications of the propeller may be introduced and the design calculation may be repeated until the optimum result is obtained. For example the following parameters of the propeller may be modified: 
$>$ the values and radial distribution of the blade skew

$>$ the values and radial distribution of the blade section profile lengths

$>$ the values and radial distribution of the blade section profile maximum thicknesses

$>$ the type of the chord-wise thickness distribution

$>$ the type of the profile mean line

$>$ the radial distribution of the hydrodynamic loading (circulation)

$>$ the number of blades

$>$ the type of duct.

The analysis of propeller operation in the non-uniform velocity field may be performed either for the propeller design condition (advance velocity and number of revolutions) or for off-design conditions without changing the propeller geometry and the velocity field. This option is convenient when compromise propellers for tugboats, fishing vessels and navy ships are designed. For these types of ships there are several different values of the operational velocity and all these conditions must be taken into account in the design process in order to obtain the optimum design.

The results of the cavitation analysis are presented in Fig. 5, while the results of calculation of the unsteady bearing forces and moments are given in Figs. 6-8.

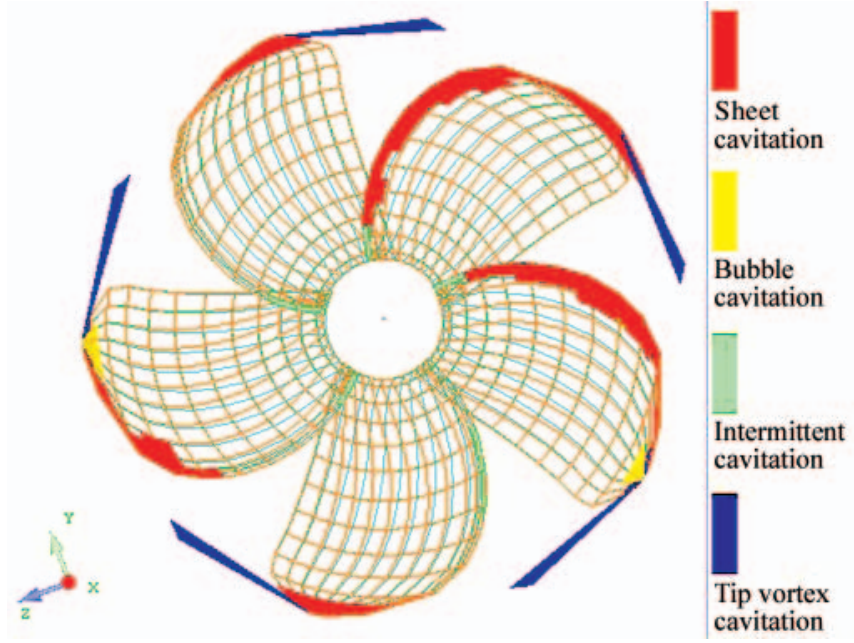

Fig. 5. An example of presentation of the different calculated forms of cavitation on the propeller blades

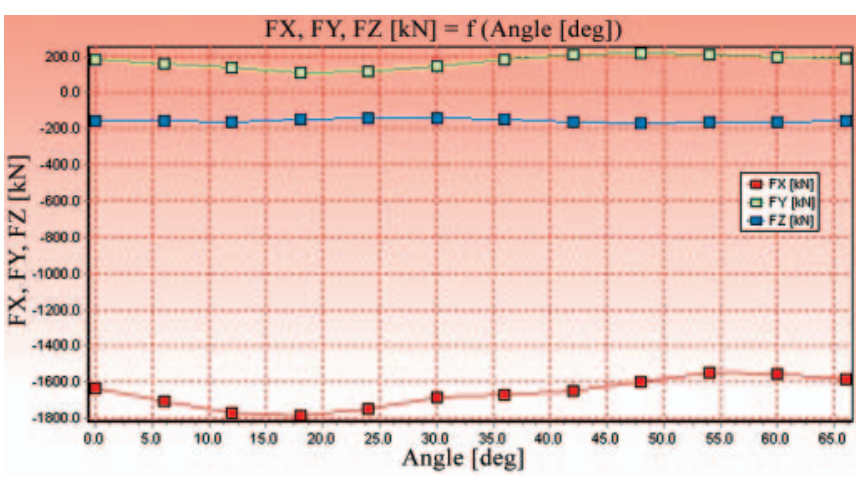

Fig. 6. The components of the calculated unsteady bearing forces for a ducted propeller

\section{COMPARATIVE ANALYSIS OF THE RESULTS}

It is generally accepted that ducted propellers demonstrate increased efficiency and operational advantages only for ships with high pull at low speed (tugboats, fishing vessels etc.). Because of that, the most widely known and applied duct is the
Wageningen 19A, which was designed specifically for high pull at low speed. In the 1970s and 1980s a series of technologically simple ducts was developed at the Institute of Fluid Flow Machinery in Gdansk [3] (cf. Fig. 2). An extensive program of model experiments was performed in order to achieve the optimum duct geometry for different operating conditions. The optimum shape of the outer and inner parts of the duct was determined and the optimum values of the diffuser angle were obtained. It was discovered that the geometry of the outer part of the duct has a strong influence on the duct resistance. The purely conical shape of this part, as in the duct 19A, may lead to almost twofold increase in the duct resistance. Fig. 9 shows the hydrodynamic characteristics of all five ducts presented in Section 2. All presented results were obtained with the same propeller.

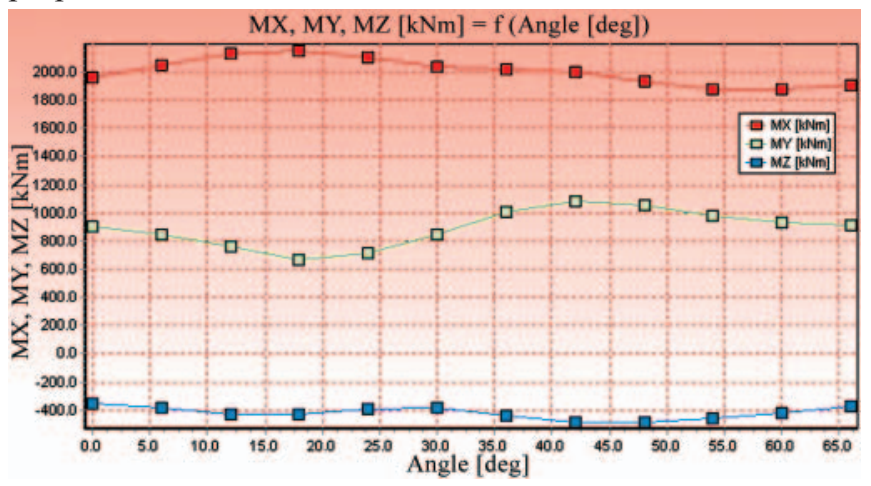

Fig. 7. The components of the calculated unsteady bearing moments for a ducted propeller

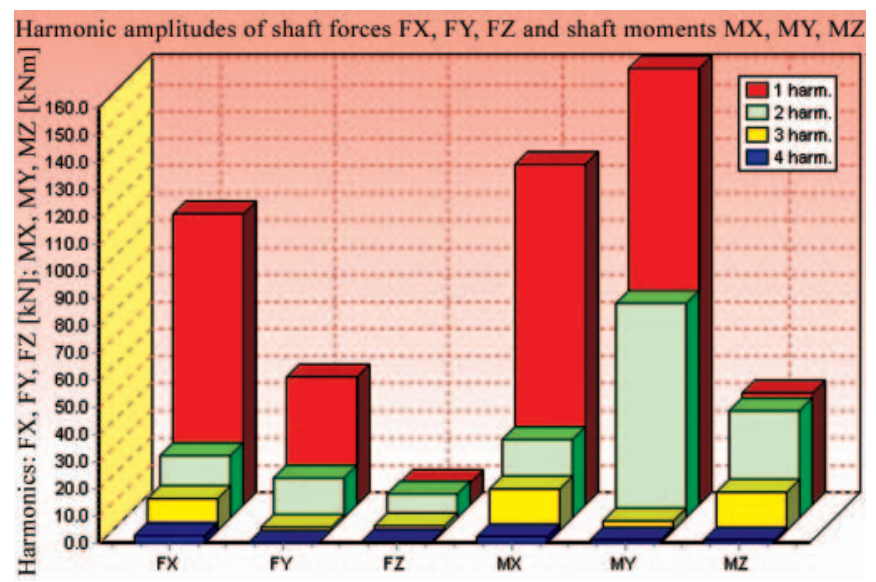

Fig. 8. The harmonic amplitudes of the bearing forces and moments for a ducted propeller

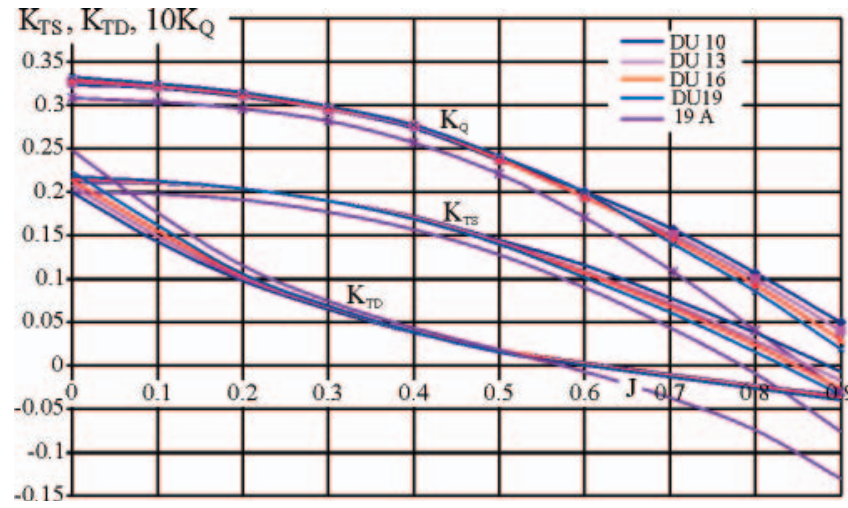

Fig. 9. The hydrodynamic characteristics of the ducted propeller for 5 ducts

As may be seen in the figure, the duct 19A has visibly higher negative thrust at high advance coefficients J. According to the research, the duct resistance coefficient CRD may be relatively 
accurately determined from the value of the duct thrust KTD corresponding to the zero propeller thrust:

$$
\mathrm{C}_{\mathrm{RD}}=\frac{\mathrm{T}_{\mathrm{RD}}}{0.5 \rho \mathrm{V}^{2} \frac{\pi \mathrm{D}^{2}}{4}}=8.0 \frac{\mathrm{K}_{\mathrm{TD}}}{\pi \mathrm{J}^{2}}
$$

The higher resistance of the duct 19A implies that it is the duct designed for high pull at low speed (in such conditions the high duct resistance ceases to influence visibly the characteristics). The relatively low resistance of the duct series DU allows them to be applied at higher values of the advance coefficient J. Fig. 10 shows the comparison of the calculated absorbed power for five ducts and for the open propeller.

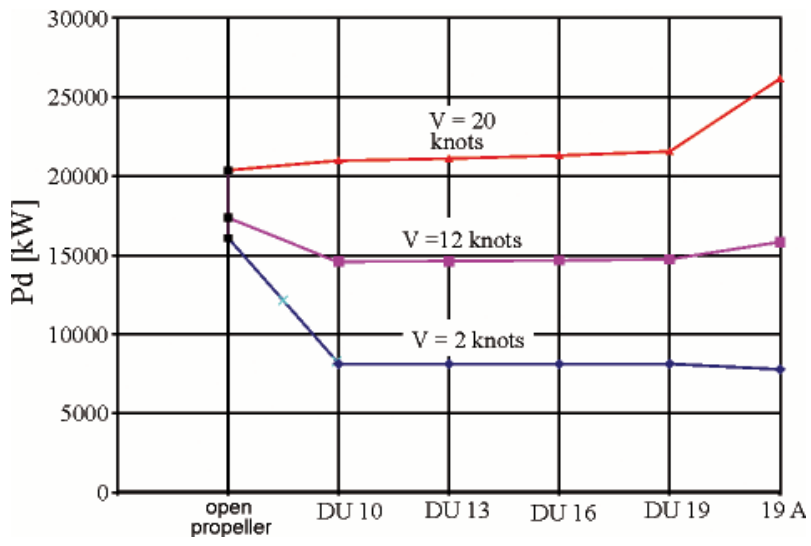

Fig. 10. Comparison of the calculated absorbed power for the ducted propellers and for the open propeller

The above described computer system was used for designing all propellers in Fig. 10 for the given rate of rotation $\mathrm{n}=120 \mathrm{rpm}$ and for the required thrust $\mathrm{T}=1500 \mathrm{kN}$. The diameter of respective propellers was determined as optimum in each case. For ducted propellers it was around $6.5 \mathrm{~m}$, and for the open propeller it was equal to $7.23 \mathrm{~m}$. The calculations were performed for three values of the inflow velocity: 2,12 and 20 knots. In order to eliminate the influence of the velocity field (which is more positive for smaller propeller diameters), all calculations were conducted for the uniform inflow.

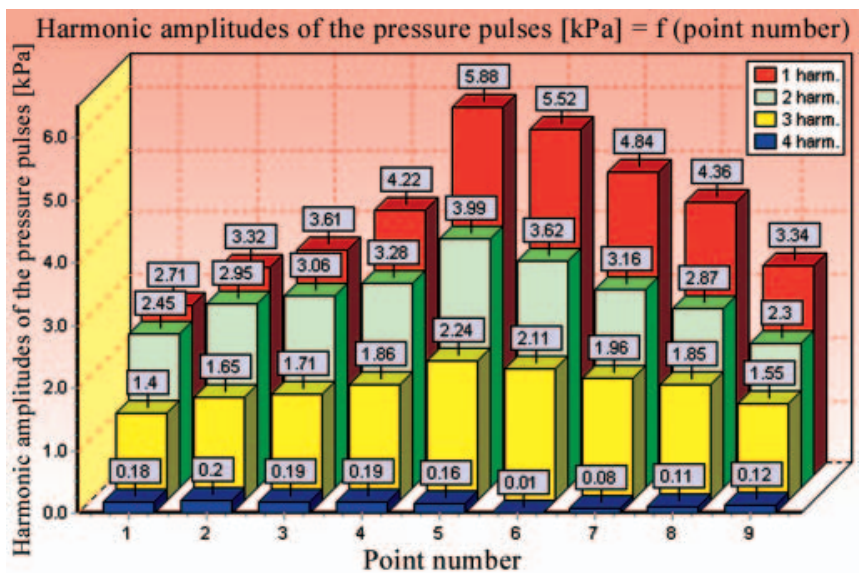

Fig. 11. Harmonic amplitudes of the induced pressure pulses for the DU13 ducted propeller

The analysis of the results presented In Fig. 10 leads to the conclusion, that as could be expected, the 19A duct performs better (i.e. absorbs less power) only at low inflow speeds. The ducts of the DU series demonstrate similar characteristics, but at low speed the duct DU19 is better, while at high speed the duct DU10 consumes less power. Very interesting conclusions may be drawn from comparison with the open propeller. Namely, only at the highest speed the open propeller is better. Both at medium and low speed the ducted propellers have a substantial advantage. Moreover, at high ship speed the ducted propellers may demonstrate other advantages: their optimum diameter is smaller and the amplitudes of the propeller-induced pressure pulses on the hull may be also smaller. Figs. 11 and 12 present the results of calculations (programs UNCA and DUNCAN) of the harmonic amplitudes of the induced pressure pulses on the hull for the ducted propeller DU13 (Fig. 11) and for the corresponding open propeller (Fig. 12). It is visible that the open propeller induces smaller pressure pulses.

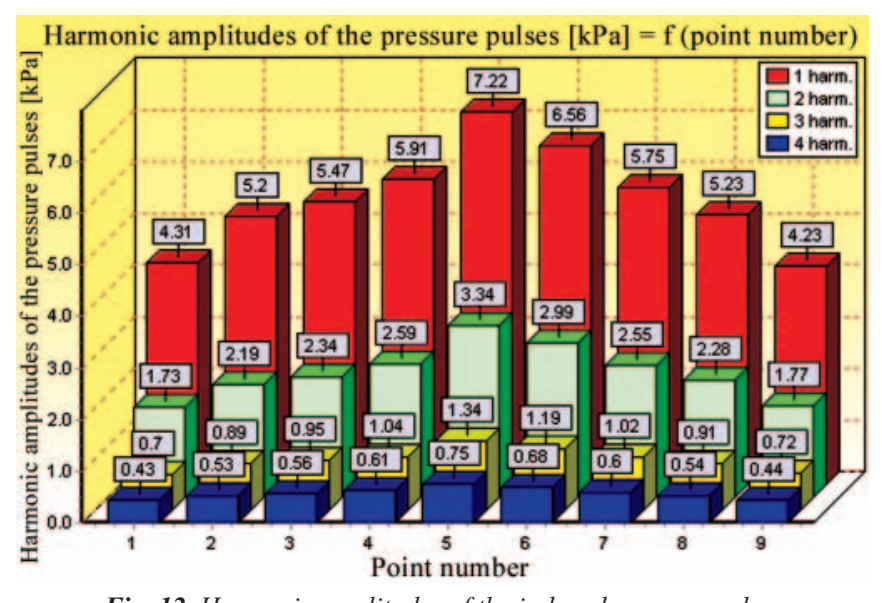

Fig. 12. Harmonic amplitudes of the induced pressure pulses for the open propeller

\section{FINAL REMARKS}

The above presented design system should significantly facilitate the process of design of ducted propellers. The system includes all components necessary in the correct process of design of propellers operating in the ducts of selected geometry (out of five options presented in Section 2.1 above). The available variants of the duct geometry enable design of high performance ducted propellers in a wide range of the operating conditions - even for relatively fast ships.

There is a widely accepted opinion that the application of the ducted propellers is advantageous only in the case of high loading and low speed (tugs, trawlers etc.). This results from the fact that in most cases only the Wageningen 19A duct was considered and this duct was designed for low speed and high bollard pull conditions.

The comparative analysis presented in the preceding Section shows, that the ducted propellers may offer competitive performance to open propellers and they may be successfully applied on ships with high speed. It should be pointed out that due to the danger of the propeller tip vortex cavitation and associated erosion on the inner surface of the duct, the Kaplan type blade outline for ducted propellers should be avoided.

\section{BIBLIOGRAPHY}

1. Koronowicz T., Krzemianowski Z., Tuszkowska T., Szantyr J.A.: A Complete Design of Ship Propellers Using the New Computer System, Polish Maritime Research No. 1 Vol. 16 (2009)

2. Koronowicz T.: The Algorithm of the Design Program Based on the Single Layer Lifting Surface for the Highly Skewed Propellers (in Polish), Report of the IFFM No. 296/86 
3. Koronowicz T.: Design of Ducted Propellers of Technologically Simple Geometry (in Polish), Proc. $5^{\text {th }}$ Symposium of Ship Hydromechanics, Gdansk 1979

4. Szantyr J. A.: A Computer Program for Calculation of Cavitation Extent and Excitation Forces for a Propeller Operating in the Non-Uniform Velocity Field, International Shipbuilding Progress, No. 296, Vol. 26 (1979)

5. Szantyr J. A.: A Method for Analysis Cavitating Marine Propellers in Non-Uniform Flow, International Shipbuilding Progress, No. 427, Vol. 41 (1994)

6. Szantyr J. A.; User Instruction for the Program DUNCAN for the Analysis of Ducted Propellers Operating in Non-Uniform Velocity Field (in Polish), Report of the IFFM No. 150/88

7. Koronowicz T., Tuszkowska T., Waberska G., Krzemianowski Z., Chaja P., Góralczyk A.: The Design Algorithm for Screw Propellers (in Polish), Report of the IFFM No. 6103/06

8. Koronowicz T., Krzemianowski Z., Tuszkowska T., Góralczyk A.: The System of Programs for Design of Ducted Propellers(in Polish), Report of the IFFM No. 345/08

9. Koronowicz T., Szantyr J.A., Krzemianowski Z., Tuszkowska T., Waberska G.: The System of Programs for Design of Ship Propellers (in Polish), Report of the IFFM No. 148/08

10.Koronowicz T., Szantyr J. A., Chaja P.: A Computer System for the Complete Design of Ship Propellers, Proc. XVII International Conference on Hydrodynamics in Ship Design, Polanica, Poland, September $19^{\text {th }}-21^{\text {st }}, 2007$
11.Koronowicz T., Krzemianowski Z., Szantyr J. A.: Numerical Determination of the Propeller Design Velocity Field Including Scale Effect and Rudder Influence, Proc. $11^{\text {th }}$ Numerical Towing Tank Symposium, Brest, France September $7^{\text {th }}-9^{\text {th }} 2008$

\section{CONTACT WITH THE AUTHORS}

Tadeusz Koronowicz, Prof. Zbigniew Krzemianowski, Ph. D. Teresa Tuszkowska, Ph. D.

Institute of Fluid-Flow Machinery, Polish Academy of Sciences Fiszera 14

80-952 Gdańsk, POLAND e-mail :ttk@interecho.com

Jan A. Szantyr, Prof.

Department of Turbomachinery and Fluid Mechanics,

Gdańsk University of Technology Narutowicza 11/12

80-958 Gdańsk, POLAND e-mail : jas@pg.gda.pl 